JINR preprint E1-98-333

Dubna 1998

\title{
Stable Algorithm for Extraction of Asymmetries from the Data on Polarized Lepton-Nucleon Scattering
}

\author{
N.Gagunashvili: \\ Joint Institute for Nuclear Research, Dubna, Russia
}

\begin{abstract}
A new algorithm for extraction of asymmetries from polarized leptonnucleon scattering data is proposed. The algorithm is stable to set-up acceptance and/or luminosity monitor acceptance variations. A statistical test for checking the data quality is proposed.
\end{abstract}

PACS: 13.88.+e, 13.60.Hb; MSC: 62-07;

key words: method, polarization, nucleon, asymmetry, spin

Dr.N.Gagunashvili,

Laboratory of Particle Physics,

Joint Institute for Nuclear Research,

Joliot-Curie str.6,

Dubna,

Moscow region,

RU-141980, Russia.

e-mail: gagunash@sunse.jinr.ru,

Tel. +70962164641

Fax. +70962165767

\footnotetext{
1 e-mail: gagunash@sunse.jinr.ru
} 


\section{Introduction}

The measurement of asymmetry in deep inelastic scattering of polarized leptons on polarized nucleons gives the main information for studying the internal spin structure of nucleons. This approach allows to avoid the problems of normalizations and, to some extend, acceptance corrections to obtain then polarized structure functions.

Usually asymmetry is evaluated according to the formula:

$$
A=\frac{1}{P^{t} P^{b}} \quad \frac{N^{+}-N^{-}}{N^{+}+N^{-}},
$$

where $N^{+}\left(N^{-}\right)$is the number of scattered leptons for the target spin parallel (antiparallel) to the beam spin orientation, $P^{t}$ is a value of target polarization and $P^{b}$ is a value of beam polarization. This formula is valid for the case when luminosity and the set-up acceptance for the parallel and anti-parallel spin orientation is the same [四]. Usually this condition takes place when two targets with the opposite spin orientation are used for measurements .

A more general formula is used in [2] where measurements are carried out with only one target, sequentially for each target spin state:

$$
A=\frac{N^{-} L^{+}-N^{+} L^{-}}{N^{-} L_{P}^{+}+N^{+} L_{P}^{-}},
$$

where $L^{+}\left(L^{-}\right)$is luminosity for each target spin state, and $L_{P}^{+}\left(L_{P}^{-}\right)$is luminosity weighted by the product of the beam and target polarization values. The formula (2) is valid if the set-up acceptance is the same and the luminosity monitor acceptance is the same for all states of the target spin.

Another method based on exact formulae for differences of cross-sections for parallel and antiparallel spin orientations is developed in [3]. According to this approach the number of events $N_{i j}$ collected for any kinematical bin and for a given pair (bin) of the beam $(i)$ and target $(j)$ polarizations, can be written as

$$
N_{i j}=\sigma_{u} L_{i j}+\sigma_{p} L_{i j} P_{i j}^{t} P_{i j}^{b},
$$

where $L_{i j}$ is the luminosity, $P_{i}^{b},\left(P_{j}^{t}\right)$ is the measured beam(target) polarization, $\sigma_{u}$ is the unpolarized part of the cross section and $\sigma_{p}$ is the polarized part of the cross section. Acceptance, dead time and other corrections are assumed to be known and properly accounted for. The unpolarized part of cross section $\sigma_{u}$ and the polarized part of cross section $\sigma_{p}$ are extracted from the relationship (3) by using the likelihood method. Asymmetry is calculated as:

$$
A=\frac{\sigma_{p}}{\sigma_{u}} \text {. }
$$

In practical cases the set-up and luminosity monitor acceptances are not known and they vary with time. It can be the cause of the asymmetry bias if the methods [2], [3] ase used. 
In this paper, we propose an algorithm for asymmetry extractions that is stable to acceptances variations. In framework of this algorithm a statistical test of the data quality check is also developed. The usage of the algorithm does not assume that acceptances are known or acceptances does not vary with time when measurements are performed.

The paper consists of 4 sections. In Sect. 2 we describe an algorithm for asymmetry extraction. An example of using the algorithm is described in Sect.3. Conclusions are given in Sect. 4.

\section{Algorithm for an asymmetry extraction}

Usually the data to determine asymmetry in lepton-nucleon interactions are collected during long periods of time, it can be done within several years. The beam and target polarization, beam intensity, luminosity, spectrometer acceptance, luminosity monitor acceptance, and other experimental conditions vary in these periods of time. To minimize the systematic errors associated with these variations, the data are divided in samples with relatively stable experimental conditions and the target polarization is frequently reversed.

The number of events $N$ collected for any kinematical bin for each sample can be written as:

$$
N=\frac{\sigma_{u} C l}{c}+A \frac{\sigma_{u} C l}{c} P^{t} P^{b},
$$

where $C$ is the set-up acceptance, $c$ is the luminosity monitor acceptance, $l$ is the measured luminosity and $P^{t}\left(P^{b}\right)$ target (beam) polarizations. The measured luminosity can be presented as:

$$
l=L c
$$

here $L$ is an unknown true value of the luminosity. For simplicity we have assumed that the dead time corrections are known and properly accounted for. We have also assumed that the target is mononuclear.

Let us define a supersample as a subset of samples following one by one in time. We also assume that for this subset the time dependence of the variable:

$$
s \equiv \frac{\sigma_{u} C}{c}
$$

can be approximated by a line

$$
s(t)=a+b t
$$

where, $a$ and $b$, are free parameters.

Assume that the set of samples can be divided in $n$ supersamples and each $i$-th

supersample, $i=1, \ldots, n$ contains $m_{i}$ samples. The number of events $N_{i j}$ collected 
for any kinematical bin and $j$-th, $j=1, \ldots, m_{i}$, sample of the $i$-th supersample, can be written as:

$$
N_{i j}=\left(a_{i}+b_{i} t_{i j}\right) l_{i j}+A\left(a_{i}+b_{i} t_{i j}\right) l_{i j} P_{i j}^{t} P_{i j}^{b} \quad .
$$

Our aim is to extract asymmetry from (9) taking into account statistical errors of $N_{i j}$, luminosity, and polarizations. The solution of this problem is found in the framework of the maximum likelihood method. The task of the maximum lakelihood procedure is to find the asymmetry, true values of luminosities $\left\langle l_{i j}\right\rangle$, target polarizations $\left\langle P_{i j}^{t}\right\rangle$, beam polarizations $\left\langle P_{i j}^{b}\right\rangle$ and parameters $a_{i}, b_{i}$.

If fluctuations of polarizations and luminosity are assumed to be Gaussian ${ }^{2}$ and for $N_{i j}$ to be Poisson, the logarithm of the likelihood functional $L$, is as follows:

$$
\begin{aligned}
-\ln L= & \sum_{i=1}^{n} \sum_{j=1}^{m_{i}}<l_{i j}>\left(a_{i}+b_{i} t_{i j}\right)+A<l_{i j}><P_{i j}^{t}><P_{i j}^{b}>\left(a_{i}+b_{i} t_{i j}\right)- \\
& -N_{i j} \ln \left(<l_{i j}>\left(a_{i}+b_{i} t_{i j}\right)+A<l_{i j}><P_{i j}^{t}><P_{i j}^{b}>\left(a_{i}+b_{i} t_{i j}\right)\right)+ \\
& +\frac{\left(l_{i j}-<l_{i j}>\right)^{2}}{2 \delta l_{i j}^{2}}+\frac{\left(P_{i j}^{t}-<P_{i j}^{t}>\right)^{2}}{2\left(\delta P_{i j}^{t}\right)^{2}}+\frac{\left(P_{i j}^{b}-<P_{i j}^{b}>\right)^{2}}{2\left(\delta P_{i j}^{b}\right)^{2}}+\mathrm{const}
\end{aligned}
$$

where $\delta l_{i j}, \delta P_{i j}^{t}, \delta P_{i j}^{b}$ are statistical errors of $l_{i j}, P_{i j}^{t}, P_{i j}^{b}$.

Minimization of Eq. (10) gives asymmetry $A$, and all other parameters $\left\langle P_{i j}^{t}\right\rangle$, $\left.<P_{i j}^{b}\right\rangle,\left\langle l_{i j}>, a_{i}, b_{i}\right.$. The complete matrix of statistical errors is calculated as the inverse matrix of the second derivative matrix of the functional (10) at its minimum [4].

Residuals examination can be applied to the quality check of the data used for asymmetry extraction [5]. They are defined as:

$$
\operatorname{Res} N_{i j}=\frac{N_{i j}-N_{i j}^{f i t}}{\sqrt{ } N_{i j}},
$$

where the fitted value of the number of events

$$
N_{i j}^{f i t} \equiv<l_{i j}>\left(a_{j}+b_{i} t_{i j}\right)-A<l_{i j}><P_{i j}^{t}><P_{i j}^{b}>\left(a_{i}+b_{i} t_{i j}\right)
$$

and

$$
\operatorname{Res} P_{i j}^{t}=\frac{P_{i j}^{t}-<P_{i j}^{t}>}{\delta P_{i j}^{t}}
$$

${ }^{2}$ There is a tendency for errors that occur in many real situations to be normally distributed due to the Central Limit Theorem. If an error is a sum of errors from several sources, then no matter what the probability distribution of the separate errors may be, their sum will have a distribution that will tend more and more to the normal distribution as the number of components increases, by the Central Limit Theorem. Thus, the assumption of normality is not unreasonable in most cases. In any case we can later check the assumption by examining residuals. 


$$
\begin{aligned}
& \operatorname{Res} P_{i j}^{b}=\frac{P_{i j}^{b}-<P_{i j}^{b}>}{\delta P_{i j}^{b}} \\
& \operatorname{Res}_{i j}=\frac{l_{i j}-<l_{i j}>}{\delta l_{i j}}
\end{aligned}
$$

The residuals plotted in the time order fluctuate around zero with the same variation and have no time trend if the quality of the data is good and choice of supersamples is reasonable.

\section{Example of the algorithm usage}

In order to demonstrate the usage of the algorithm, pseudodata were simulated. We take asymmetry $A$ equal to $0.1, \sigma_{u}=1$. number of samples $n$ equal to 80, luminosity $\bar{L}_{i}, i=1, \ldots, 80$, polarizations $\bar{P}_{i}^{t}, \bar{P}_{i}^{b}$, acceptances $C_{i}$ and $c_{i}$ as they are shown in Fig. 1, Using formulae (5) and (6), $\bar{N}_{i}$ and $\bar{l}_{i}$ are calculated.

The measured number of events $N_{i}$ is simulated as Poisson distributed random variable with a mean value equal to $\bar{N}_{i}$. The measured values of luminosity $l_{i}$ and polarization $P_{i}^{t}, P_{i}^{b}$ were obtained by adding fluctuations to the luminosity $\bar{l}_{i}$ and polarization $\bar{P}_{i}^{t}, \bar{P}_{i}^{b}$ values. Fluctuations were simulated as Gauss distributed random variables with the mean value equal to zero and $\sigma$ equal to $2 \%$ for luminosity, $0.5 \%$ for target polarization and $5 \%$ for beam polarization. These pseudodata are shown in Fig. 2.

The algorithm was used for processing the pseudodata divided into a different number of supersamples with the same number of samples. The result of the algorithm application is shown in Table 1. The last row of Table 1 presents the asymmetry extracted with the algorithm from [2].

In order to calculate the average value of the asymmetry and bias, the pseudodata described above were simulated 25000 times. The average value for the asymmetry $<A>$ and bias defined as the difference between the asymmetry average value and its true value 0.1 are also shown in Table 1 . As follows from Table 1., the algorithm proposed in this article gives an essentially lower bias than the algorithm in [2].

Residuals were analysed to choose the optimal number of supersamples. This analysis has shown that the number of supersamples can be chosen equal to 8 , residuals for this case are shown in Fig. 3. $N_{i}$ and its fitted value $N_{i}^{f i t}$ are also shown in Fig. 3. 
TABLE 1. Results of the asymmetry extraction for different numbers of supersamples. The $\chi^{2}$ are calculated for the number of events $N_{i}$ and their fitted value $N_{i}^{f i t}$. The last row presents the results of the algorithm [2] application.

\begin{tabular}{|l|l|l|l|l|}
\hline $\begin{array}{l}\text { Number of } \\
\text { supersamples }\end{array}$ & $A$ & $\chi^{2}$ & $<A>$ & Bias \\
\hline \hline 1 & $0.0799 \pm 0.1426$ & 89.4987 & $0.1015 \pm 0.0009$ & $1.5 \%$ \\
\hline 2 & $0.0810 \pm 0.1426$ & 88.8028 & $0.1016 \pm 0.0009$ & $1.6 \%$ \\
\hline 4 & $0.0679 \pm 0.1430$ & 81.8742 & $0.0995 \pm 0.0009$ & $-0.5 \%$ \\
\hline 8 & $0.0584 \pm 0.1442$ & 63.0929 & $0.1008 \pm 0.0009$ & $0.8 \%$ \\
\hline 16 & $0.0676 \pm 0.1451$ & 38.4059 & $0.1007 \pm 0.0009$ & $0.7 \%$ \\
\hline \hline Algorithm [2] & $0.1112 \pm 0.1456$ & & $0.1160 \pm 0.0009$ & $16.0 \%$ \\
\hline
\end{tabular}

\section{Conclusions}

The algorithm for extraction asymmetry from the polarized lepton-nucleon scattering data stable to the set-up and luminosity monitor acceptances is proposed. In framework of the developed algorithm a statistical test for checking the data quality is proposed. The algorithm has no restrictions related with small statistics of experimental events and can be applied for inclusive as well as for semi-inclusive physics. Principles of constructing the algorithm can be used in any other field of the experimental particle and nuclear physics.

\section{Acknowledgements}

The author would like to thank G.I.Smirnov for critical reading of the manuscript and useful discussion and A.A.Fechtchenko for encouragement of this work.

\section{References}

[1] SMC, D.Adams et al., Phys.Rev. D 56 (1997) 5330.

[2] HERMES, K.Ackerstaff et al., Phys.Lett. B 404 (1997) 383.

[3] N.D.Gagunashvili et al, Nucl.Instr. and Meth. A 412 (1998) 146.

[4] Y.Bard, Nonlinear Parameter Estimation, Academic Press New York, 1974.

[5] N.R.Draper, H.Smith, Applied Regression Analysis, Johm Wiley \& Sons, New York, 1981. 

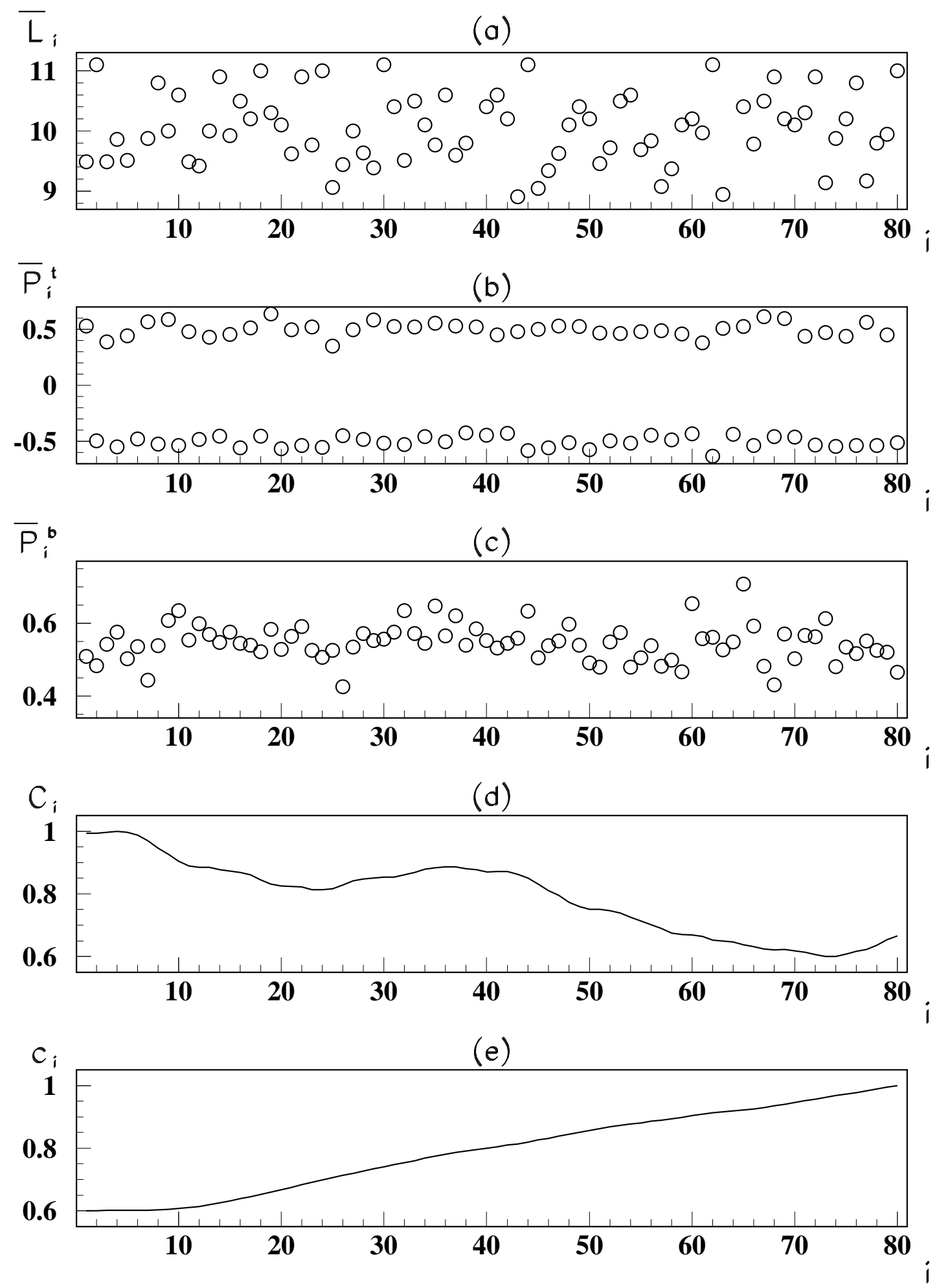

Fig.1. The data for simulation of pseudodata: (a) luminosity, (b) target polarization, (c) beam polarization, (d) set-up acceptance, (i) luminosity monitor acceptance. 

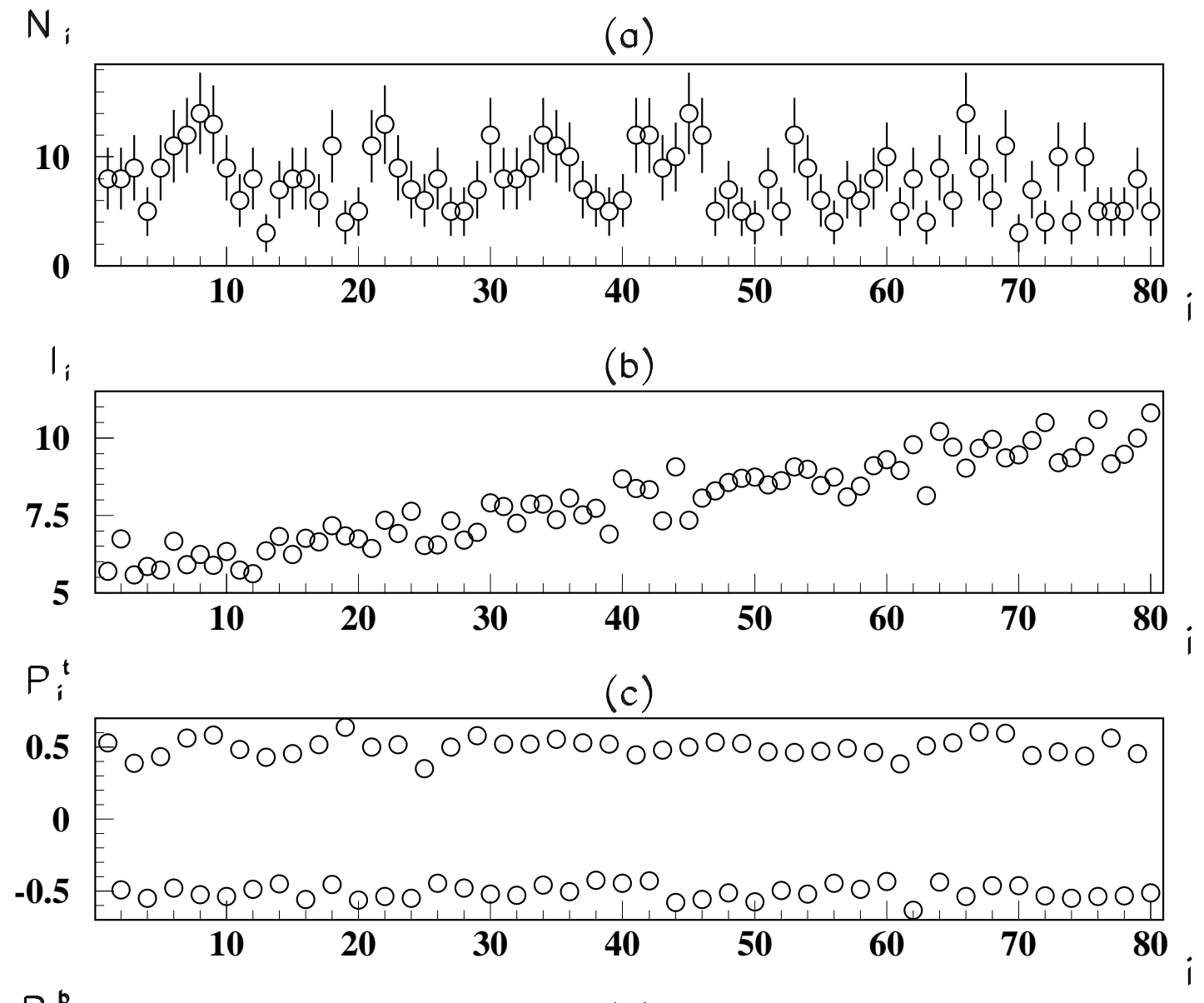

$P_{i}^{b}$

(d)

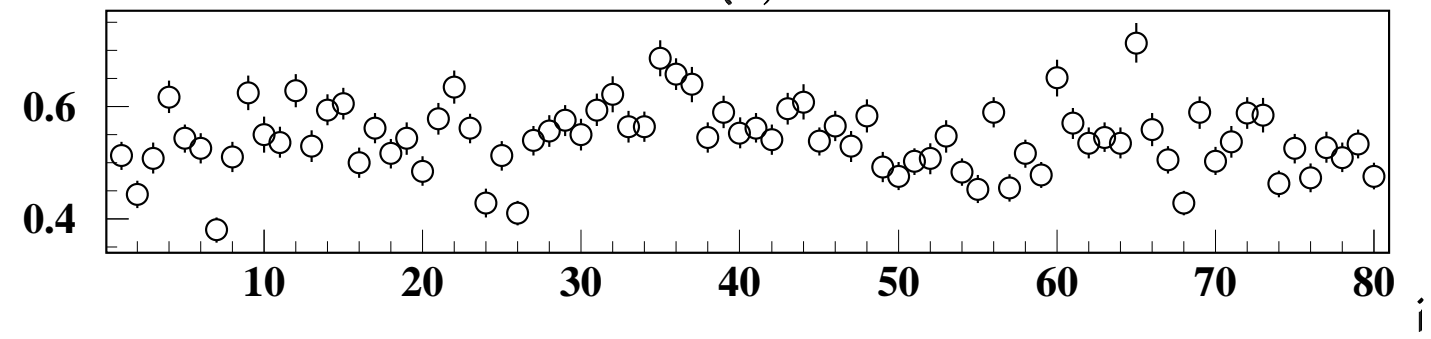

Fig.2. The pseudodata: (a) measured number of events, (b) measured luminosity, (c) measured target polarization, (d) measured beam polarization. 
Res $N_{i}$

(a)

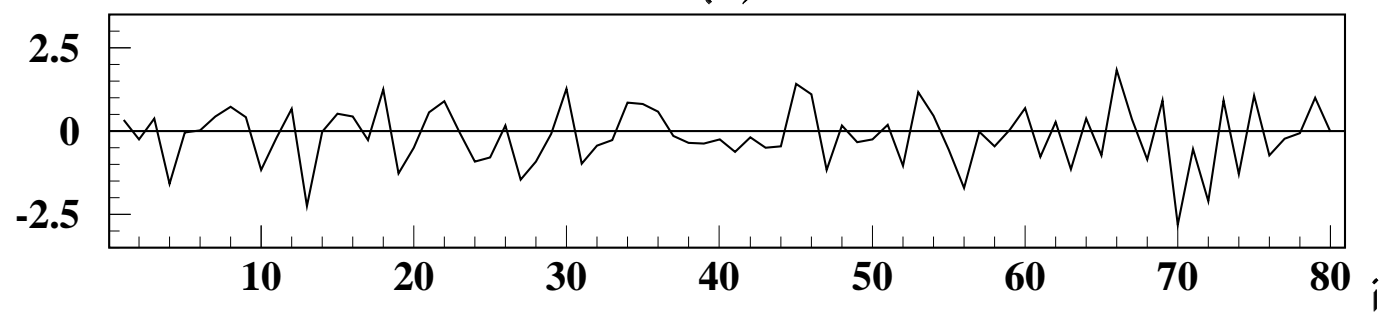

Res I;

(b)

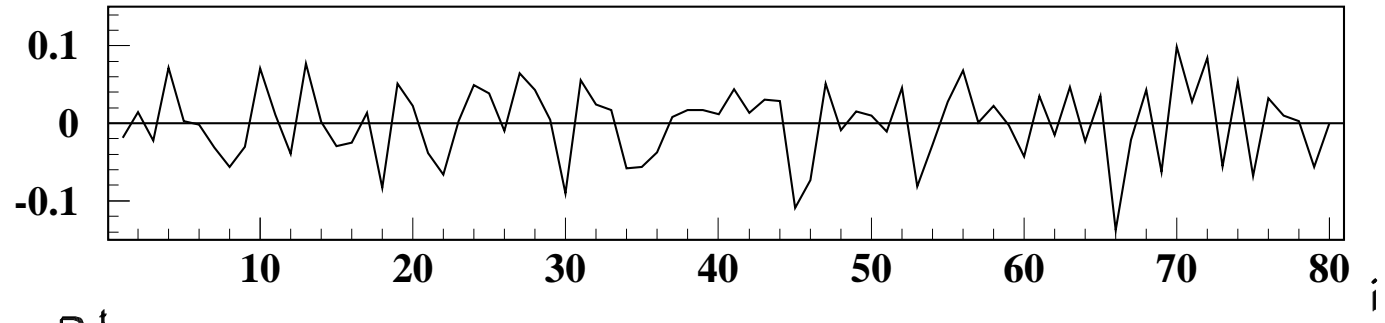

$\operatorname{Res} P_{i}^{t}$

(c)
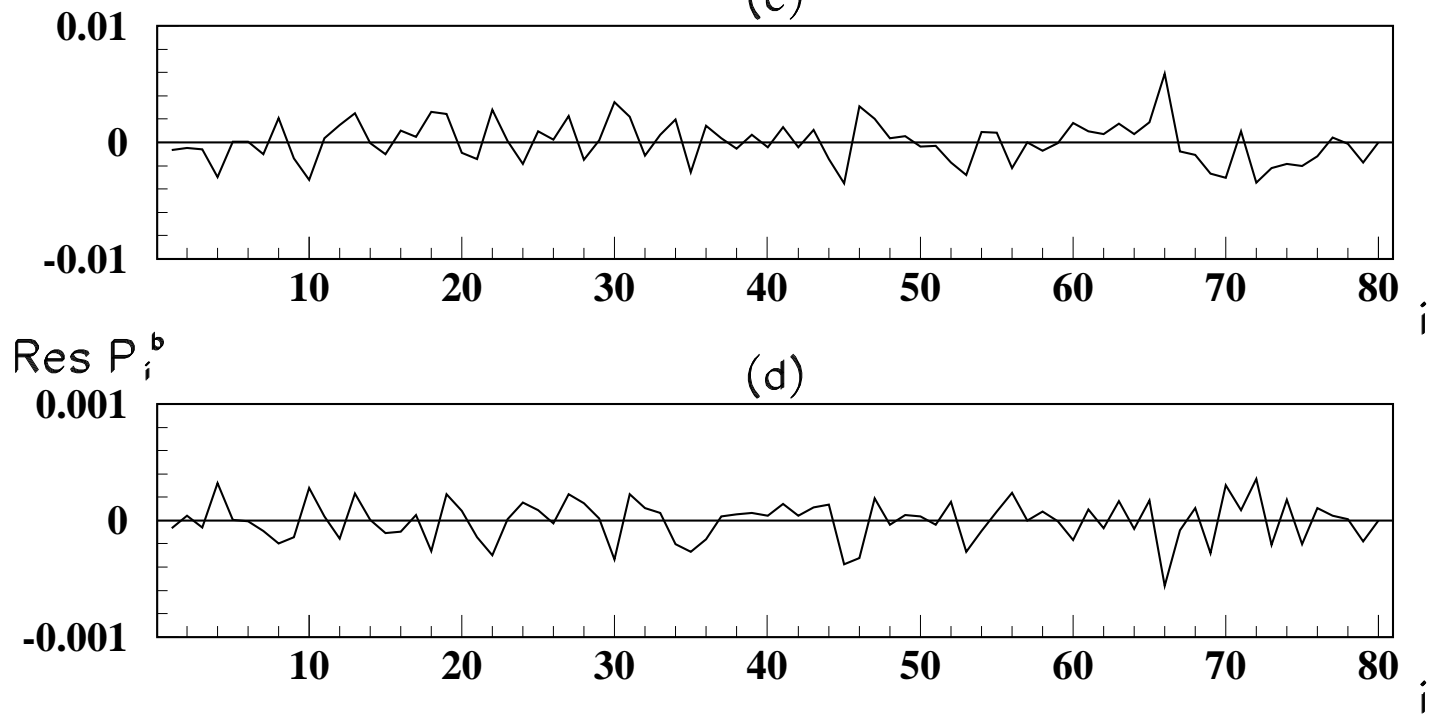

Fig.3. Residuals (a) for number of events, (b) for luminosity, (c) for target polarizations, (d) for beam polarizations. 


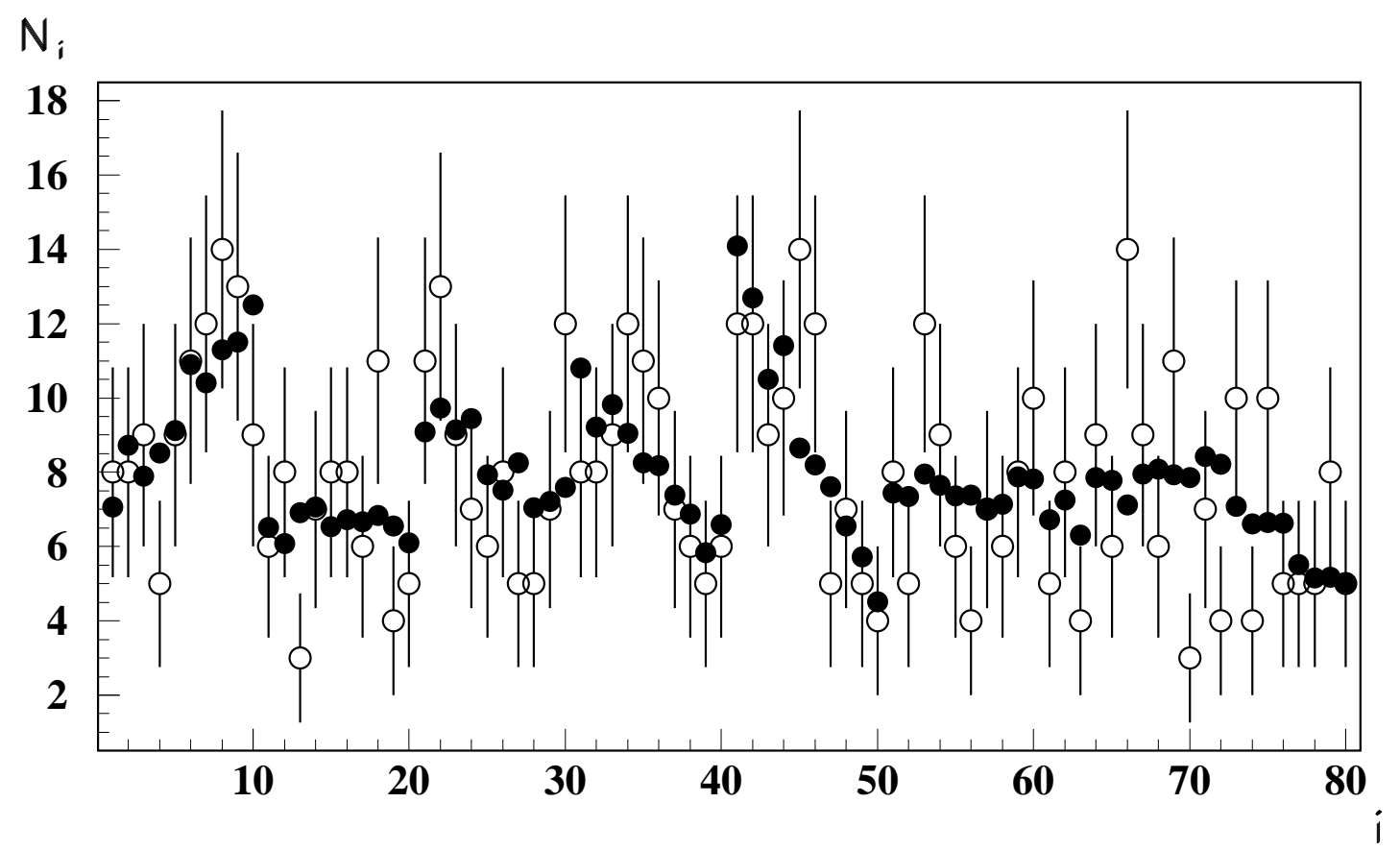

Fig.4. The number of events for samples $N_{i}$ and their fitted values $N_{i}^{f i t}$ (close cycles). 